\title{
Reparación de imagen y comportamiento prosódico: entre la atenuación y la intensificación
}

Face repair and prosodic behavior: between mitigation and intensification

GLORIA UCLÉS RAMADA

UNIVERSITAT DE VALÈNCIA / GRUPO VAL.ES.CO

ADRIÁN CABEDO NEBOT

UNIVERSITAT DE VALĖNCIA / GRUPO VAL.ES.CO

RESUMEN: Este estudio analiza, en el marco de la conversación española peninsular, la prosodia, vinculada a la reparación lingüística de la imagen; tradicionalmente la reparación ha sido asociada al ámbito de la atenuación. Para este objetivo se han buscado muestras de reparación en el corpus de conversaciones coloquiales Val.Es.Co y en la transcripción del programa de telerrealidad Gandía Shore. Con esta base de datos se ha realizado un análisis fonético usando el programa Praat y, posteriormente, los datos extraídos se han visualizado en grupos mediante la aplicación de dos técnicas estadísticas: el análisis múltiple de correspondencias y el análisis de conglomerados. Los resultados obtenidos señalan que la reparación de imagen no es un fenómeno demasiado frecuente $\mathrm{y}$, además, que los rasgos fónicos que lo acompañan pueden aparecer atenuados o intensificados en una proporción similar.

Palabras clave: atenuación, reparación, intensificación, prosodia, imagen.

ABSTRACT: This study analyses -in a corpus of Spanish informal conversationsthe prosody of fragments where there has been a linguistic face repair. Traditionally the concept of repair has been linked with mitigation. In order to do so, repair fragments in corpus of informal conversations Val.Es.Co and in a selftranscribed corpus of the reality TV show Gandía Shore have been compiled. With these data, a phonetic analysis with Praat has been carried out and, subsequently, the outcome has been plotted in two groups using two statistical tests: multiple correspondence analysis and cluster analysis. The results show that face repair is a rather infrequent phenomenon and the phonetic traits associated can be mitigated or reinforced in a similar proportion.

Keywords: mitigation, repair, intensification, posody, face. 


\section{INTRODUCCIÓN}

En esta investigación se analizan ocurrencias de reparación discursiva procedentes de conversaciones españolas peninsulares; al mismo tiempo, el interés se sitúa en la correlación de estos casos con una prosodia atenuada o intensificada. Cabe mencionar, en tal sentido, que la reparación discursiva se ha vinculado tradicionalmente con el fenómeno pragmático de la atenuación (Albelda Marco et al., 2014; Benoit, 1997; Briz Gómez y Albelda Marco, 2013); aun así, a partir de un conjunto de conversaciones en español, se pretende observar si el nivel fónico, no siempre explorado en estudios pragmáticos, solo corresponde a una minimización expresiva o si, por el contrario, puede haber reparaciones vinculadas a situaciones de énfasis. Véase, como ejemplo, el caso de (1):

(1) 14 MJ: ¿y de Ximo?/ ¿qué?// ¿qué tal?

15 M: ¿Ximo? ¿Ximo $\downarrow$ Ximo?/ ¿quién es Ximo?

16 MJ: quién es Ximo $\downarrow /$ eel- el de gafas $\rightarrow$

17 M: jese es el que te estoy hablando!§

18 MJ: § ;aah!/ ¿XIIMOO $? \S$

19 M: $\S$ sí§

20 MJ: $\S$ es que como me has dicho Juanjo $\uparrow$

21 M: ;ah! ;bueno!/ perdón $\downarrow / /$ eel Ximo

(Cabedo Nebot y Pons Bordería, 2013¹)

En este fragmento, la agresión a la imagen de MJ no procede de un improperio ni de un acto de menosprecio, sino de un cuestionamiento sobre la relación de las palabras de MJ con el contexto en el que se enmarcan; en este ejemplo dos participantes, MJ y M, conversan sobre un chico que gusta a M. En la intervención 15 («¿Ximo? ¿Ximo $\downarrow$ Ximo?/ ¿quién es Ximo?») M pone en duda el conocimiento de MJ sobre la persona de la que se está hablando, hasta que esta indica («eel- el de gafas») y, finalmente, se hace eco del error de, en este caso, M («es que como me has dicho Juanjo $\uparrow »)$. En este momento, M se da cuenta de su equivocación y subsana esta agresión a la imagen de MJ, en la intervención 21, con un («ah $\downarrow$ ¡bueno!/ perdón $\downarrow / /$ ee/ Ximo»). En esta secuencia, la subsanación no solo es verbal, sino que se acompaña fónicamente de tonos descendentes marcados («ah $\downarrow /$ perdón $\downarrow »)$ y de expresiones enfatizadas («iah!, ¡bueno!»), junto con otros factores como la proliferación de pausas y de una vacilación en forma de alargamiento («ee»). Se trata, por tanto, de una reparación marcada o enfatizada de la imagen discursiva de la hablante MJ.

Como se observa en el ejemplo anterior, la preservación de la imagen social del individuo supone un elemento importante para cualquier interacción. La imagen se entiende, en su definición tradicional, como «the positive social value a person effectively claims for himself by the line others assume he has taken during a particular contact» (Goffman, 1967: 5). La imagen del individuo, por lo tanto, se articula como una construcción interactiva, una representación cognitiva del yo que se negocia, en el marco de una conversación, durante los sucesivos intercambios comunicativos (Benoit, 1997; Hernández Flores, 2004, 2015; Spencer-Oatey, 2007). Para el español existe bibliografía que incluye actividades lingüísticas dirigidas a cuidar, preservar o restituir la imagen de los hablantes (Figueras Bates, 2018; Gómez Sánchez, 2004; Hernández Flores, 2004, 2015; Villalba, 2016); la imagen, en todo caso, se entiende en este estudio como «constructo multidimensional que abarca tanto componentes sociales, culturales, situacionales, relacionales como individuales» (Figueras Bates, 2018: 278-79).

\footnotetext{
${ }^{1}$ Conversación 23, Intervenciones 14-21.
} 
La reparación de la imagen dañada de un participante durante una conversación habitualmente conlleva dos elementos relacionados. En primer lugar, uno de los hablantes se responsabiliza de un acto de habla y, al mismo tiempo, este acto se considera ofensivo para la imagen de otro participante (Benoit, 1997). La bibliografía no arroja un consenso sobre la tipología de reparaciones que pueden realizarse, más allá de las disculpas, justificaciones o ensalzamientos positivos (Compton, 2016; Gómez Sánchez, 2004; Hernández Flores, 2004; Vela Delfa, 2016); en general, «when an FTA has already been committed various measures are put in place to deal with this like, outright denial of not being responsible for the FTA or shifting blame, evasion of responsibility, reducing offensiveness, making a corrective action and even mortification» (Asiko Ambuyo, Ngesa Indede y Ndichu Karanja, 2011: 211).

Así pues, en tanto en cuanto se aprecia una voluntad de restituir una imagen ajena, la reparación de imagen se asocia también con frecuencia con la cortesía. Algunos autores van más allá y la incluyen directamente como una de las funciones de la atenuación lingüística que, en tal sentido, puede ir orientada por el hablante para generar cortesía (Briz Gómez y Albelda Marco, 2013: 303). Es asimismo en esta aproximación al fenómeno en la que cobra especial importancia el contexto comunicativo, sin el que no pueden entenderse todos los casos en los que, como en la atenuación, el valor pragmático no se vincula necesariamente a una forma, sintagma $u$ enunciado, sino que puede extenderse globalmente por varias de estas unidades.

Así mismo, la atenuación y la intensificación son dos recursos pragmáticos que permiten enfocar la imagen social de los hablantes durante el transcurso de una conversación. Se entiende atenuación como la «minimización de la fuerza ilocutiva y del papel de los participantes en la enunciación para lograr llegar con éxito a la meta prevista y que es utilizada en contextos situacionales de menos inmediatez o que requieren o se desea presenten menos inmediatez comunicativa» (Briz Gómez y Albelda Marco, 2013: 292). Por su parte, la intensificación es una «estrategia comunicativa por la que se refuerza algún elemento del enunciado o de la enunciación con el fin de conseguir un objetivo determinado en la comunicación y, en general, destinada al acuerdo o desacuerdo en la conversación» (Albelda Marco, 2005, 2007). Una de las hipótesis de este estudio es que tanto atenuación como intensificación pueden ser recursos usados por el hablante para restituir la imagen dañada de otros participantes - presentes o ausentes- durante la conversación.

En esa misma línea explicativa, aunque es considerada importante, no se ha incidido demasiado en la vinculación entre el mecanismo prosódico y algunos fenómenos pragmáticos, como la atenuación o la intensificación (Hidalgo Navarro, 2009, 2015; Rojas Avendaño, Blondet Serfaty y Álvarez Muro, 2014). Esta combinación es relevante en tanto que permite evidenciar formalmente la intención del emisor sobre la imagen de interlocutores presentes o ausentes en la conversación.

Precisamente por esta globalidad del fenómeno de reparación, el estudio fónico no puede circunscribirse a unidades mínimas como la palabra o el grupo fónico (Quilis, 1981), sino que requiere de unidades más amplias; es, en tal caso, donde la unidad del paratono cobra mayor importancia (Brown, Currie y Kenworthy, 1985; Wichmann, 2000).

En concreto, un paratono funciona como un párrafo delimitado por factores prosódicos (intensidad o tonalidad elevadas, pausas amplias) que, al mismo tiempo, marca cambios de tópico dentro de la secuencia discursiva en la que se inserta (Brown \& Yule, 1983). Se trata, por tanto, de un grupo de entonación o más de uno que pueden estar unidos por un fenómeno de supradeclinación (Wichmann, 2000); sobre todo, esta macrounidad entonativa parece estar delimitada por unas fronteras tonales marcadas: 
«one or more IUs ending in a major (terminal) final boundary, where any (optional) previous IU carries a minor (continuing) boundary tone» (Izre'el y Mettouchi, 2015: 30); $\mathrm{y}$ «the most obvious phonetic cues are the high placing of the onset of a paratone, the brevity of pauses within it, and the gradual drift down in overall pitch height towards a low ending». (Brown 1990, 92). En cuanto a la relación con el significado que estas unidades transportan, algunos autores hablan incluso de paratonos mayores y de paratonos menores, según el grado de informatividad transmitido (Brown, Currie y Kenworthy 1985,71$)$.

En relación con la expresión fónica de la atenuación y de la intensificación, son constantes las referencias generales al tono o a la intensidad de la voz para acompañar ambos fenómenos pragmáticos. Más concretamente, «el efecto atenuante se obtiene mediante la reducción de la amplitud de la voz (a veces hasta el susurro)» (Hidalgo Navarro, 2009: 20). Este ascenso o descenso de la prominencia fónica, se concreta a veces en patrones tonales específicos; este es el caso de algunas variantes del español, como sucede en el habla de Mérida (Venezuela), en la que se detecta un patrón $\mathrm{H}^{*}+\mathrm{L}$ (subida tonal en la sílaba tónica más un descenso inmediato) y una duración de las sílabas que duplica la de aquellas que no expresan atenuación (Rojas Avendaño, Blondet Serfaty y Álvarez Muro, 2014). También en el español peninsular se ha encontrado una configuración circunfleja para expresar atenuación (Hidalgo Navarro, 2009).

La intensificación, por su parte, puede asociarse en ocasiones a estructuras exclamativas o enfáticas (Hidalgo Navarro, 2009); otros rasgos que se mencionan son el de tonema ascendente, alargamiento e inflexiones melódicas singulares a lo largo de la secuencia discursiva en la que aparecen (Albelda Marco, 2004).

Enunciados sin ninguna marca lingüística de intensificación, gracias a la entonación, se pueden intensificar, puesto que la curva entonativa es un elemento de significación. Por otro lado, aunque no es el único factor, la entonación es fundamental para entender la intensificación de algunos fenómenos mencionados anteriormente

(Albelda Marco, 2007: 83)

En este trabajo, por tanto, se pretende observar en qué medida la reparación de la imagen social de los hablantes se restituye (o se intenta) mediante el uso de estrategias fónicas que minimizan o enfatizan la prosodia de las secuencias discursivas sobre las que se proyectan. Todo ello se realizará mediante un estudio de corpus conversacional (sección 2.1) y a través de técnicas estadísticas de visualización de datos (sección 2.3).

\section{METODOLOGÍA}

La metodología de este estudio aúna una perspectiva cuantitativa y una perspectiva cualitativa. En realidad, la interpretación contextual de los datos recogidos se ha sistematizado posteriormente mediante técnicas estadísticas de visualización para, en un último apartado, servir de entrada a la constitución de un número determinado de categorías que puedan tener relevancia lingüística. De esta manera, la estadística utilizada es un medio (Glynn, 2010, 2014b) y no un fin en sí mismo; esto es, el hecho lingüístico siempre es el foco principal. Es así como el monismo metodológico asociado a ciencias experimentales (López Serena, 2011) se sortea en beneficio del objeto interpretativo propio de estudios pragmáticos que, necesariamente, se vinculan a un contexto y a la interpretación de este. 


\subsection{CORPUS}

Se han detectado 26 casos de reparación lingüística de imagen tras haber recogido, leído y analizado 14 capítulos del programa televisivo Gandía Shore y 7 conversaciones coloquiales del corpus Val.Es.Co 2.0 (Cabedo Nebot y Pons Bordería, 2013). Todos ellos contienen el español peninsular como lengua vehicular. El desajuste entre muestras se justifica porque solo se han seleccionado las conversaciones del corpus Val.Es.Co. 2.0 que manifiestan una calidad de audio óptima, mientras que la totalidad de material procedente de Gandía Shore presenta una muy buena calidad. Es este también el motivo por el que se ha combinado material sonoro procedente de grabaciones secretas y de formato televisivo, dada la insuficiencia de archivos sonoros con calidad procedente de los corpus conversacionales.

El programa Gandia Shore, grabado y proyectado en televisión por la cadena MTV entre 2012 y 2013, recoge las vivencias de ocho jóvenes (cuatro chicas y cuatro chicos) durante el periodo veraniego en una playa de Gandía (Valencia). Este formato combina secuencias audiovisuales en las que los hablantes conversan en un entorno físico determinado (una casa, la playa, el trabajo...) con secuencias en las que son entrevistados individualmente y en las que relatan situaciones o acciones acontecidas en un tiempo cercano al momento de la grabación. Los 14 capítulos del reality Gandía Shore componen un total de 53423 palabras para el género conversacional; cabe tener en cuenta que no se computan en ese total las palabras del género entrevista.

Por su parte, las 7 conversaciones coloquiales analizadas del corpus Val.Es.Co. 2.0 contienen un total de 16708 palabras.

La transcripción y los datos acústicos se han recogido directamente desde ELAN (Max Planck Institute, 2017) y desde PRAAT (Boersma y Weenink, 2017), respectivamente.

\subsection{VARIABLES INCLUIDAS EN EL ESTUDIO}

Las variables usadas en este estudio han sido las siguientes:

1. Origen. Esta variable recoge la tipología del causante de la reparación. Las categorías han sido el emisor, otro hablante presente u otro participante ausente.

2. Imagen. Se trata de la imagen afectada por la reparación. Las dos categorías de esta variable han sido la imagen de un receptor presente o la de un receptor ausente.

3. Reparación. Los tipos de reparación recogidos en este estudio han sido mayoritariamente dos: la disculpa y la justificación. Se ha incluido una tercera categoría llamada otros para aquellos casos en los que el investigador no ha tenido una certeza clasificatoria.

4. Prosodia. Las variables prosódicas han partido de una variable global, llamada prosodia, en la que aparecen dos valores fundamentales: atenuada o énfasis. Estos valores proceden del registro de valores tonales y de intensidad.

5. Finalmente, la especificación de la variable prosodia se subdivide en cuatro variables: modulación, intensidad, velocidad y alargamiento. Todas ellas incluyen los valores sí o no. 
En cuanto a las variables prosódicas utilizadas, las de tonalidad/intensidad y velocidad de habla han sido recodificadas desde variables numéricas a variables categóricas para poder realizar una de las pruebas estadísticas (análisis múltiple de correspondencias). En cuanto a la tonalidad/intensidad, los valores críticos de ascenso o descenso significativo se han situado en los 3 st para la tonalidad y en los 30-60 dB para la intensidad; esto es, en aproximadamente el doble de los valores críticos establecidos por bibliografía precedente (Fernández Planas et al., 2002; Manchón, 2011; Marrero Aguiar, 2008; Roseano y Fernández Planas, 2013).

\subsection{PRUEBAS ESTADÍSTICAS REALIZADAS}

En esta investigación se han aplicado dos pruebas estadísticas usando Factominer (Lê, Josse y Husson, 2008), paquete del programa de análisis estadístico R (R Development Core Team, 2017); concretamente, las pruebas utilizadas han sido el análisis múltiple de correspondencias y el análisis de conglomerados.

El análisis de correspondencias (simple [AC] o múltiple [AMC]) es una técnica estadística que proyecta la relación entre las variantes en un gráfico con forma de mapa. Este mapa, con dos ejes, expone la cantidad de varianza explicada en las dos primeras dimensiones detectadas por el método estadístico. Por su parte, las dimensiones distribuyen a las categorías según la capacidad de estas para agruparse y, por ende, para caracterizar a un determinado grupo de elementos (Greenacre, 2007).

Esta prueba (AMC) es también adecuada para casos en los que no hay un amplio número de registros, dado que no es prueba con validez de contraste poblacional. En otras palabras, la prueba apunta relaciones que solo un mayor número de frecuencias podría dotar de significatividad estadística. Para subsanar esta limitación, la prueba puede completarse con la aplicación de una chi-cuadrado para observar la dependencia o independencia entre las variables utilizadas (Glynn, 2014b, 2014a).

Finalmente, un análisis de conglomerados ha permitido observar mejor los grupos de individuos que se pueden detectar a partir de las cercanías y lejanías establecidas por el análisis múltiple de correspondencias.

\section{RESULTADOS}

Los resultados de esta investigación proceden del análisis descriptivo de los 26 ejemplos recogidos en la base de datos; del uso de técnicas de visualización multivariante, como el análisis múltiple de correspondencias; y de la posterior agrupación de elementos, a través del análisis de conglomerados.

\subsection{FRECUENCIAS ABSOLUTAS Y RELATIVIDAZAS}

Como puede observarse en la Tabla 1, no se han detectado manifestaciones reparadoras de la imagen en todos los archivos analizados. De hecho, de 14 episodios de Gandía Shore y de 7 conversaciones del corpus Val.Es.Co. solo se encuentran instancias de reparación en 12 de ellos: 
Tabla 1. Frecuencias absolutas y relativas de la base de datos utilizada

\begin{tabular}{|l|r|r|r|l|r|r|r|r|r|r|r|r|l|}
\hline Archivo & C1 & C2 & C3 & G2 & G5 & G6 & G9 & G13 & G3 & G4 & G8 & G11 & $\begin{array}{l}\text { C= conversación / } \\
\text { G= Gandía Shore }\end{array}$ \\
\hline Palabras & 2287 & 3000 & 1610 & 3182 & 3224 & 3490 & 3454 & 4067 & 2797 & 4377 & 3880 & 3282 & $\begin{array}{l}\text { Suma total: } \\
38650\end{array}$ \\
\hline $\begin{array}{l}\text { Frec. } \\
\text { Reparación }\end{array}$ & 3 & 4 & 1 & 3 & 1 & 1 & 1 & 1 & 2 & 5 & 1 & 3 & Suma total: 26 \\
\hline $\begin{array}{l}\text { Frec. Reparac. } \\
\text { por millón }\end{array}$ & 1311 & 1333 & 621 & 942 & 310 & 286 & 289 & 245 & 715 & 1142 & 257 & 914 & Media: 698 \\
\hline
\end{tabular}

Lo que puede deducirse de la anterior tabla es que la reparación de imagen no es un fenómeno frecuente en español hablado. De hecho, en otro estudio de género discursivo similar como la entrevista (Albelda Marco, 2013), se ha detectado una frecuencia relativa de 309 casos de reparación por millón de palabras. En concreto, de 960 actos de habla procedentes de 24 entrevistas sociolingüísticas, solo se encontraron 103 casos de reparación de la imagen de los hablantes. En línea similar a lo observado en la Tabla 1, puede señalarse que, en general, en cada conversación o entrevista hay una media de entre 1 a 4 actividades de reparación.

Otros estudios particulares han prescindido directamente de la reparación como una función característica de la atenuación y se han circunscrito al análisis de la protección de la imagen propia y de la ajena (Figueras Bates, 2018).

\subsection{DATOS DESCRIPTIVOS DE LA VARIABLE DE ATENUACIÓN O INTENSIFICACIÓN FÓNICA}

Antes de comentar los valores descriptivos de cada variable, se parte en este apartado del análisis de la variable prosodia, que recoge la tonalidad/intensidad global del paratono. De los 26 registros de la base de datos, hay 14 casos de prosodia atenuada y 16 casos de énfasis; no hay, por tanto, una distribución distinta de estas categorías para la variable prosodia $(\mathrm{X} 2[1]=0.13$, valor $\mathrm{p}=0.84)$. Este valor negativo, sin embargo, tiene una consecuencia teórica evidente ya que pone de manifiesto que la reparación de la imagen del hablante no utiliza únicamente procedimientos de atenuación, sino que también se sirve de expresiones de intensificación. Se trataría de énfasis fónicos que aparecen como en 2:

(2) Ar: pero porque ella intentaba decirle algo

C: que Ylenia tiene este carácter pero no es mala ¿me entiendes? y punto es así y punto y se acabó

C: que pasa que no perdonas ¿no? o sea la gente la caga porque es humana y no perdonas

C: escucha

L: me gusta hacer las cosas muy bien y dejar las cosas

C: escucha te estoy diciendo

L: clara tengo mi personalidad tengo un carácter un poco fuerte

C: claro que sí claro que sí

L: vale pero soy un bellísima persona

(Gandía Shore, episodio 4)

En (2) la hablante C pretende interceder ante L por Y, la persona responsable del daño a la imagen de $\mathrm{L}$ ausente en la conversación. Llama la atención que la misma hablante cometa dos actos de habla contradictorios: una reparación de la imagen de Y y un ataque a la imagen de L. En primer lugar, la reparación de la imagen de Y se realiza mediante un énfasis fónico notable, con ascenso de más de $30 \mathrm{~dB}$ y de 3 st en relación con la media de la hablante $\mathrm{C}$ («que Ylenia tiene este carácter pero no es mala ¿me entiendes? y punto es así y punto y se acabó»)); en segundo lugar, esta reparación 
intensificada se combina, al mismo tiempo, con una agresión a la imagen de L por parte de la misma hablante $\mathrm{C}$ («que pasa que no perdonas ¿no? o sea la gente la caga porque es humana y no perdonas»).

Por lo tanto, uno de los datos que más interés provoca es el de esta variable general prosodia. Debe recordarse que, tradicionalmente, la reparación se ha asociado en la bibliografía precedente como una de las funciones de la atenuación (Briz Gómez y Albelda Marco, 2013). Los datos encontrados en este estudio sugieren que el énfasis fónico (una de las marcas de la intensificación pragmática) puede vincularse en determinadas situaciones contextuales con la reparación de una imagen dañada.

\subsection{DATOS DESCRIPTIVOS DE LAS VARIABLES FÓNICAS}

Si se presta atención al resto de variables fónicas, cruzadas también en este caso con la variable prosodia, se llega al gráfico de la Figura 1.

Figura 1. Gráficos de barras que representan las frecuencias de las variables prosódicas
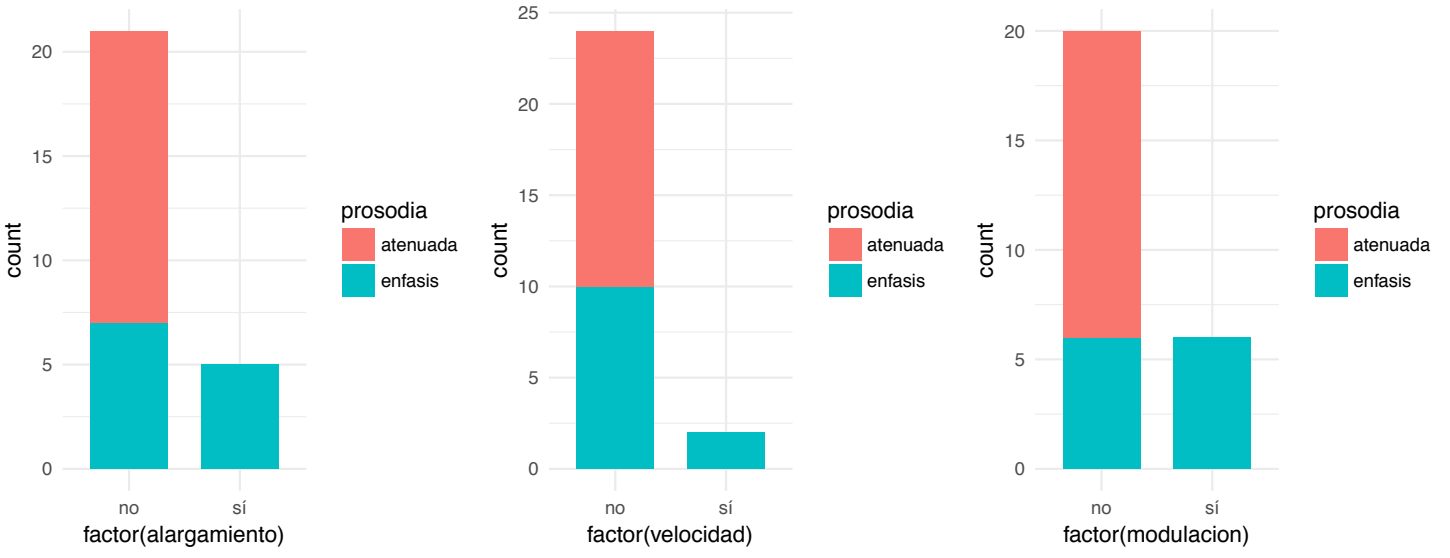

En el gráfico anterior se proyectan los valores de las variables prosódicas individualizadas. En general, los casos en los que la prosodia aparece enfatizada se corresponden en no demasiados casos con alargamientos ( 5 de 26), velocidad de habla ( 2 de 26) o modulación tonal (6 de 26). Por lo que se refiere a las relaciones estadísticas significativas, solo la variante prosodia enfatizada está acompañada de modo significativo por alargamiento fónico o modulación entonativa; en estos casos, a partir de una prueba de chi-cuadrado, se han observado los residuos de Pearson que, cuando son superiores a 1.96, implican una relación significativa entre las categorías analizadas (Moore y McCabe, 1999).

Por el contrario, del gráfico también se deduce un factor relevante: los casos de intensidad baja (prosodia atenuada) no han coincidido en este estudio con ninguna de las otras variables fónicas. Se trata de un hecho llamativo ya que algunas referencias bibliográficas previas (Hidalgo Navarro, 2009; Rojas Avendaño, Blondet Serfaty y Álvarez Muro, 2014) sí han encontrado correlaciones de variables fónicas para la expresión de otras funciones de la atenuación, como las que suponen la prevención de la imagen del emisor o del interlocutor. En esta investigación al menos, por tanto, la prosodia atenuada no se ha reflejado mediante procedimientos de alargamiento, velocidad de habla o modulación entonativa, sino únicamente mediante descenso del tono o de la intensidad global del paratono. 


\subsection{DATOS DESCRIPTIVOS DE LAS VARIABLES NO FÓNICAS}

En cuanto a las variables no directamente fónicas, en la Figura 2 puede observarse la distribución de los registros en tres de las variables que constituyen la base de datos analizada (origen discursivo de la reparación, imagen agredida y tipo de reparación); cada variable se ha cruzado, además, con la variable prosodia. Ninguno de estos cruces ha resultado estadísticamente significativo, con valores $\mathrm{p}$ superior a 0.05 en todos ellos para la prueba chi-cuadrado.

Figura 2. Gráficos de barras que representan las frecuencias de las variables no prosódicas del estudio
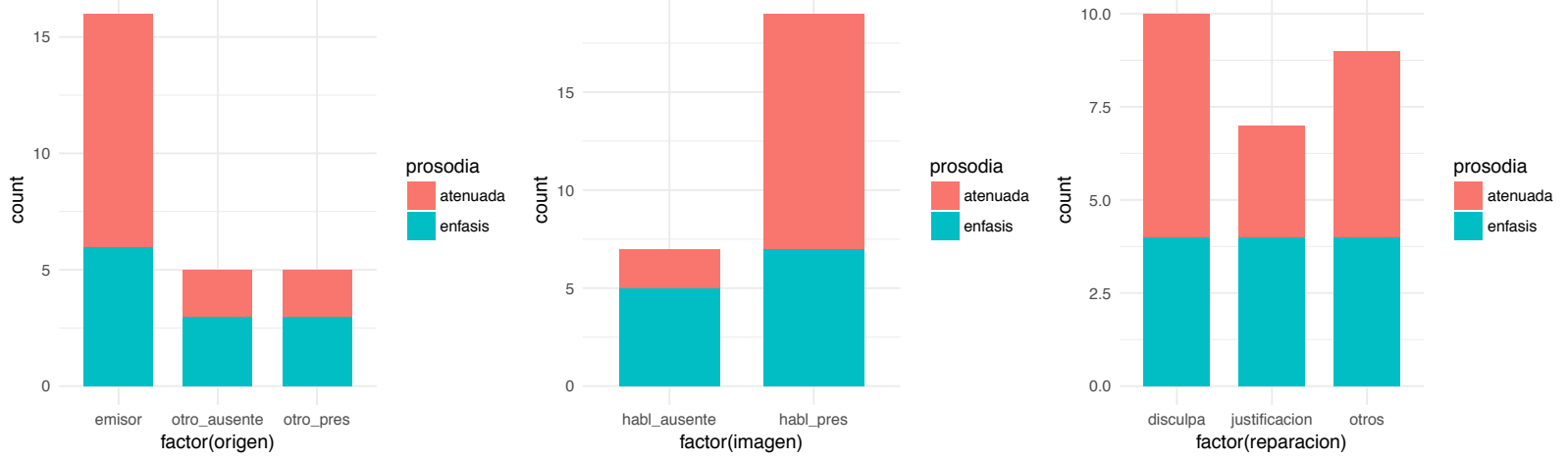

Llama la atención, para la primera variable, que, en 16 de 26 casos, el emisor de la reparación sea, al mismo tiempo, el causante de la afrenta a la imagen de otro participante. Por su parte, las imágenes reparadas suelen proceder en gran medida de hablantes presentes en el contexto, con 19 de 26 casos. En estos dos casos, el hablante suele utilizar con mayor frecuencia una intensidad o tono bajos.

Finalmente, la tipología de las reparaciones recoge un mayor número de disculpas (10 de 26), mientras que la categoría de otros, aquella que recoge elementos que no se habían sabido clasificar a priori, constituye un amplio número de registros (9 de 26). Como se verá más abajo (sección 4.5), esta dificultad para clasificar algunos ejemplos queda parcialmente resuelta después de haber realizado un análisis de conglomerados.

\subsection{ANÁLISIS MÚLTIPLE DE CORRESPONDENCIAS}

La prueba ACM refleja la relación entre los registros de una base de datos y, tal y como se evidencia en la Figura 3, proyecta sobre esa agrupación las variantes más frecuentes que se acercan para cada grupo. En aras de facilitar la lectura del mapa bidimensional, los análisis con ACM suelen excluir del gráfico los individuos o números de registro de la base de datos y dejan solo la representación de las variantes. Posteriormente, el análisis de conglomerados, observable en la Figura 4 de la sección 3.5, expone la unión de los individuos en grupos con características compartidas. 
Figura 3. Mapa bidimensional extraído mediante el análisis múltiple de correspondencias

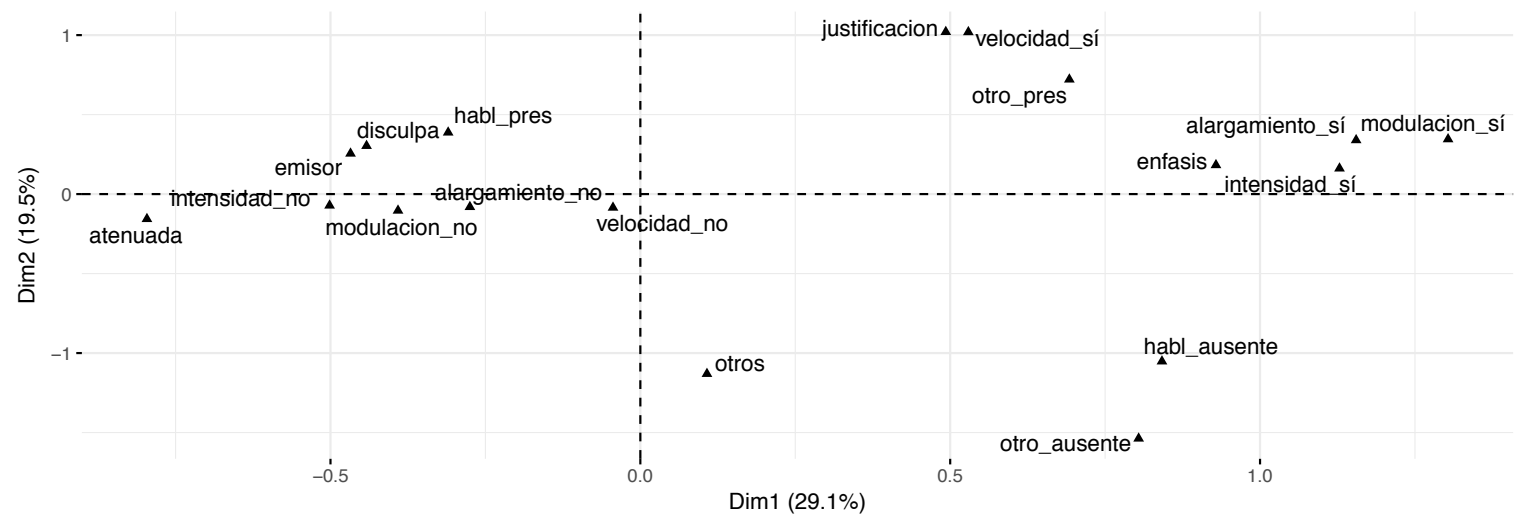

Para poder interpretar correctamente la relación entre las categorías hay que tener en cuenta las dimensiones que arroja el análisis; en tal sentido, «each dimension of a multivariate analysis can be described by the variables (quantitative and/or categorical). These variables can have participated to the construction of the factorial axes (they can be active or supplementary)» (Lê, Josse y Husson, 2008: 8). Por lo tanto, una dimensión se entiende como un conjunto de variables y variantes que explican un porcentaje de variación de los datos y, por tanto, de agrupaciones posteriores de los registros en la base de datos.

El paquete Factominer dentro del software de procesamiento estadístico R permite medir la varianza de los datos explicados por cada una de estas dimensiones. En esta investigación, la primera dimensión explica el 29.1\%; la segunda dimensión, el 19.5\%; finalmente, la tercera dimensión el $16.1 \%$. En total, casi el $70 \%$ de la variabilidad de los datos de este estudio se explican con las primeras tres dimensiones.

Como conjunto de datos, cada dimensión privilegia unas variables y unas variantes concretas frente a otras:

1. En la primera dimensión, puntúan con valores altos las variantes fónicas (modulación, alargamiento, énfasis...), también los casos en los que tanto el causante como el receptor de la agresión a la imagen no están presentes en el contexto comunicativo.

2. En la segunda dimensión puntúan alto un causante de la agresión a la imagen distinto del emisor de la reparación y, como tipo de reparación, la justificación.

3. En la tercera dimensión, que no aparece proyectada en el mapa, puntúa de forma elevada una velocidad de habla alta y, como en la segunda dimensión, como causante de la reparación, otro participante presente.

\subsection{ANÁLISIS DE CONGLOMERADOS: GRUPOS DE REPARACIONES DE IMAGEN}

Más abajo, en la Figura 4, se observa la distribución de los 26 registros de la base de datos de este estudio. El procedimiento de análisis de clúster, extraído mediante Factominer y dispuesto visualmente mediante la librería Factoextra, establece 3 grupos diferenciados. En general, los análisis de agrupación, como este mismo, proceden de un análisis múltiple de correspondencias previo, por lo que las agrupaciones de datos, en general, tienden a vincularse con las categorías previamente comentadas (Lê, Josse y Husson, 2008). 


\section{Figura 4. Visualización del análisis de clúster en el que se proyecta la relación entre los registros de la base de datos}

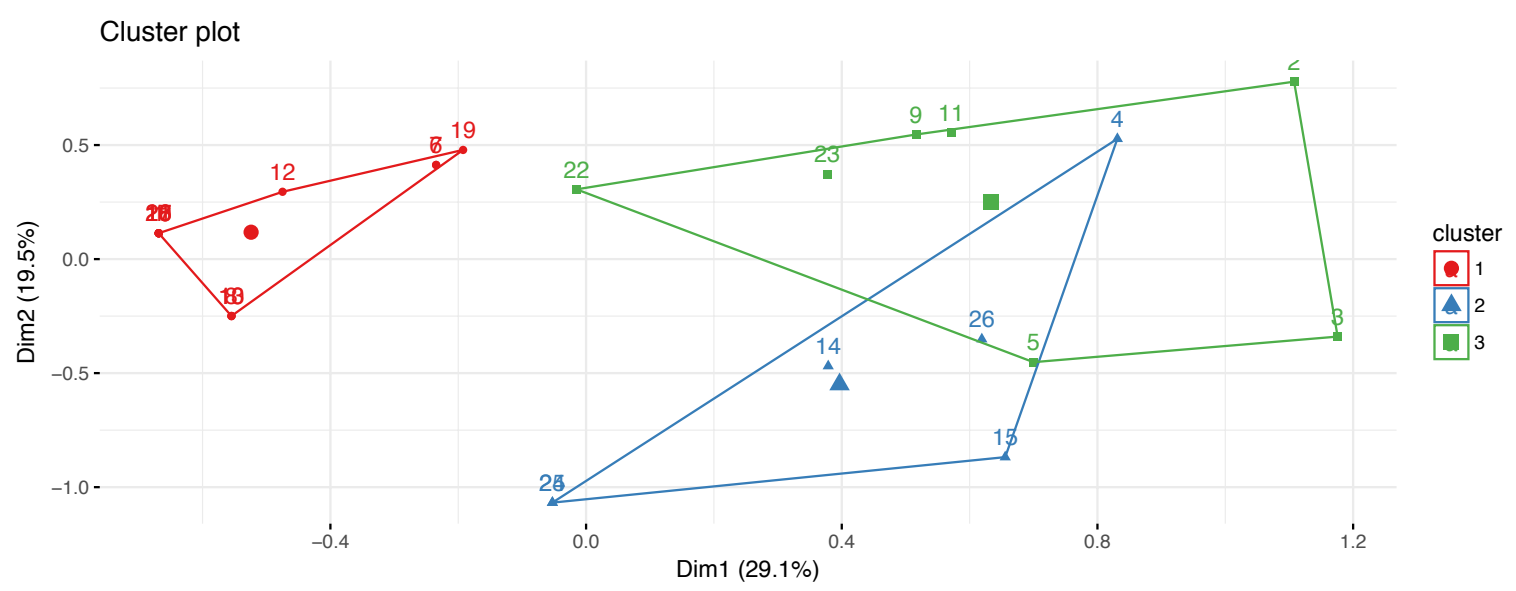

En las líneas que siguen se comentarán las características de cada uno de estos grupos a partir de ejemplos concretos. Al mismo tiempo, como se observa en la Figura 4, hay algunos pocos casos en los que se muestra una intersección; se trata, en síntesis, de algunos casos catalogados previamente por los investigadores como otra categoría de reparación distinta a la disculpa o a la justificación. En estos casos, el análisis de clúster proyecta un solapamiento con el grupo dominado por la categoría de justificación.

\subsubsection{Primer grupo}

En el primer grupo, compuesto por 13 casos de 26, se observa la mayor concentración de registros. En él destaca la disculpa; así mismo, el emisor de la reparación es también el responsable de la amenaza a la imagen de un oyente que, por su parte, se encuentra presente. Se trataría de un caso como el que se ejemplifica en (3):

(3) L: Gata ven un momento ¿puedo hablar contigo?

G: ¿ahora? dime ¿qué pasa?

L: nada que siento lo que pasó l'otro día ¿vale? que siento lo que pasó l'otro día en casa

Ar: ooh

G: esperaba que me dijeras algo

Ar: bien abracito

G: porque yo creo que te pasaste bastante

L: en verdad m'arrepiento y yo qué sé estaba mosqueao

G: encima porque ibas ya pero es que te puedes meter conmigo todo lo que tú quieras tío pero con mi madre sabiendo que l'has conocido y tal

L: de los errores s'aprenden ¿no?

G: vale m'alegro por ayudarte

(Gandía Shore, episodio 11)

Las manifestaciones de disculpa en (3) son evidentes. No solo la situación comunicativa motiva la reparación ( $L$ se acerca para pedir perdón a $G$ sobre un comportamiento pasado), sino que las propias formas lingüísticas de L son prototípicas de una reparación de imagen («siento lo que pasó l'otro día ¿vale? que siento lo que pasó 
l'otro día en casa»). En los casos de disculpa, como también muestra este mismo ejemplo (3), se acompañan habitualmente de un descenso de la prominencia tonal o de intensidad.

\subsubsection{Segundo grupo}

El segundo grupo está compuesto por 6 casos de 26. Sus categorías más importantes son la justificación como tipo de reparación, el causante de la agresión a la imagen está presente pero no es el mismo que emite la reparación y, finalmente, hay una ligera cercanía con la prosodia enfatizada, mediante una variada gama de recursos fónicos (alargamiento, modulación y, sobre todo, velocidad de habla). Como muestra, puede observarse el siguiente ejemplo (4):

(4) Ab: Que tampoco te rayes ahora. Pero aquí están tus amigas, aquí están tus amigas, pues ponte tu copita con tus amigas a bailar y ya está. Si no hay problema. No llores. Que no llores. ¿Por qué te pones a llorar ahora?

Y: ¿Qué te pasa? ¿Por qué lloras? ¿Por qué lloras, tía? Que estamos en plan broma, riéndonos.

Ab: Si no ha pasado nada.

Y: No has quedado mal ni nada, ¿vale? Te soltamos eso porque nos da rabia que nos hayáis vacilado y ya está. No se van a pensar que eres una guarra ni nada, ¿vale?

(Gandía Shore, episodio 2)

En el ejemplo (4), hay dos participantes, $\mathrm{Ab}$ e Y, que intentan restaurar la imagen de una participante presente, $X$; esta piensa que su imagen ha sido agredida por comentarios de otros hablantes y, también, que su comportamiento ante esa situación puede haber provocado la mofa o el rechazo entre sus interlocutores. Tanto Ab como Y exponen justificaciones sobre la agresión realizada; estas, por un lado, giran en torno al concepto de broma («que estamos en plan broma, riéndonos») y, por otro lado, restauran la imagen de la participante al minimizar las repercusiones de su reacción previa («si no ha pasado nada», por parte de Y) y restar importancia al contenido verbal concreto de la agresión realizada («No se van a pensar que eres una guarra ni nada, ¿vale?»).

\subsubsection{Tercer grupo}

El tercer grupo lo componen 7 casos de 26. En él se incluyen registros en los que no se ha sabido catalogar inicialmente el tipo de reparación o, al menos, se han observado dificultades para catalogarlos como disculpas o justificaciones. Este grupo, además, lo forman casos en que el emisor de la agresión no está presente durante el intercambio comunicativo o, también, por reparaciones de la imagen de un participante ausente.

(5) C dice que no dormiré

$\mathrm{C}$ vale va

A (RISA)

B café solo

B ¿tocadito de Terry o sin tocar?

C no hay tarry tarry no hay ahí

$\mathrm{B}$ ¿tú quieres cortado Ainoa?

A no yo (i(desde)) cuando tomo café hija mía?

B hija mía

B pues no sé a lo mejor te da un fuu

A me da taquicardias 
En el caso de (5), A se siente agredida porque en el entorno familiar hay consolidado un conocimiento compartido de que no le gusta el café; por eso, reacciona negativamente («no yoo/ desde cuando tomo café hija mía?») ante la oferta de la hablante B («¿tú quieres cortado Ainoa?»). Esta, en la siguiente intervención, intenta restituir la imagen de A («hija mía/ pues no sé a lo mejor te da un fuu»). Ni la producción oral de B ni el contexto en el que se produce hace entender este fragmento como una disculpa o como una justificación de la emisión realizada; se trata más bien de una respuesta incoherente dentro del marco de conocimiento compartido entre A y B. Básicamente, incumpliendo las máximas de cantidad y calidad (Grice, 1991), B no responde explícitamente a la pregunta de A y tampoco justifica el motivo de su ofrecimiento. Como mucho, el «hija mía» exclamado por $\mathrm{B}$ sí puede entenderse más como una emisión cercana a la disculpa, aunque no de modo directo.

Así mismo, como se observaba en la Figura 4, hay una zona de intersección entre el grupo 3 y el grupo 2. Dado que el segundo grupo ocupa un espacio de conglomerado mayor, este integra a parte del tercer grupo y no en dirección inversa. De este modo, algunos de los casos en los que se observaban dudas pueden clasificarse, también, como pertenecientes al segundo grupo. Se trataría de casos como el siguiente:

(6) E: ¿Quién es?

B: Soy Belén.

E: Ooye, ¿qué pasa? Antes era un amigo.

(Gandía Shore, episodio 2)

Uno de los participantes en el programa, L, se hace pasar por otro participante, E y llama por teléfono a B; las palabras de L no son especialmente halagadoras hacia B. Al poco tiempo, B llama a E y este intenta reparar la ofensa realizada. Este es un ejemplo en el que se observa énfasis fónico, velocidad de habla alta y alargamiento vocálico, por lo que se acerca más al grupo 2, que quedaba delimitado por una ligera proximidad con las variables fónicas. Es, en este sentido, en el que quizá tiene sentido entender el ejemplo (6) como una justificación ya que, grosso modo, se protege la imagen de la agredida justificando que, bajo el amparo de la amistad, el emisor de la ofensa ha actuado con una cierta licencia que se espera que sea entendida por la persona ofendida.

\section{CONCLUSIONES}

En esta investigación se han analizado 26 casos de reparación discursiva de la imagen. En tal sentido, la reparación comporta el que un hablante intente restituir la imagen social de otro hablante ya que esta pueda haber sido dañada por alguna intervención previa. En general, se han encontrado algunos resultados interesantes.

Los casos de reparación discursiva de la imagen resultan ser bastante escasos en la conversación española peninsular; de este modo, cada 15 minutos (duración media de las conversaciones analizadas en esta investigación) se encuentran entre 1-5 casos de reparaciones; estas, al mismo tiempo, suelen ir asociadas con valores de aumento o disminución fónica.

En términos de frecuencia relativizada, la reparación, de media, se encuentra en uno 300-500 casos por millón de palabras. El contraste es evidente con otros resultados relacionados con la preservación de la imagen propia o ajena en entornos conversacionales; por ejemplo, algunos estudios han observado unos 4000 casos por millón de palabras para defensa de la imagen propia y 6500 casos por millón de palabras para la preservación de la imagen ajena (Albelda Marco, 2013; Estellés Arguedas y 
Cabedo Nebot, 2017; Figueras Bates, 2018). Debe advertirse que la frecuencia relativa se ha obtenido a partir de los datos de frecuencia, subtotales y totales de palabras ofrecidos en las obras de los autores mencionados. La intención de esta relativización ha sido únicamente la de marcar cuán poco frecuente es la reparación de imagen en comparación con su protección, bien sea propia o ajena.

La reparación discursiva puede utilizar tanto mecanismos de atenuación como de intensificación/énfasis de la prosodia, representados normalmente mediante ascensos o descensos globales de tono o intensidad en el paratono donde se encuentra la estrategia verbal de reparación. De este modo, se rechaza la hipótesis nula por la que la reparación de imagen se vincula únicamente con casos de atenuación pragmática (Briz Gómez y Albelda Marco, 2013; Compton, 2016) y, de este modo, se consolida como una función discursivo-social de primer orden que, según la naturaleza del contexto comunicativo, puede presentarse fónicamente atenuada o intensificada.

Otro dato relevante extraído a partir de la base de datos, es que en la conversación española peninsular quien causa una agresión a la imagen de otro interlocutor suele reparar esta mediante una prosodia atenuada y a través disculpas formalmente expresadas (lo siento, perdón...); por su parte, si quien agrede no es quien finalmente pretende la restitución de la imagen, la reparación se realiza con justificaciones lingüísticas y utilizando una intensidad o tonalidad altas, también acompañada en ocasiones de otras fórmulas fónicas como los alargamientos vocálicos, la velocidad de habla o las inflexiones tonales singulares.

En otro orden de cosas, las técnicas estadísticas aplicadas han permitido observar tres grupos que se diferencian por la mayor influencia de algunas variables frente a otras. En un primer grupo, las disculpas, como tipo de reparación, se vinculan con un emisor que, al mismo tiempo, es el responsable de la amenaza previa a la imagen del interlocutor; por la parte fónica, la disculpa se acompaña de valores bajos de tono o intensidad.

En un segundo grupo, se encuentran las justificaciones, con valores altos de tonalidad o intensidad y una posible presencia de otros factores fonéticos como, entre otros, alargamientos vocálicos o una mayor velocidad de habla. En paralelo, estas restituciones se relacionan con un causante de la amenaza a la imagen que está presente, pero que curiosamente no es el mismo emisor de la reparación.

Finalmente, en un tercer grupo se catalogan casos en los que no se advierte claramente una justificación o una disculpa; estos registros se asocian con la imagen restituida de una persona no presente en la conversación y, también, con emisores de la amenaza no presentes. Son, por ejemplo, casos en los que alguien explica a otro hablante una situación que involucra a otras dos personas, es decir, son sobre todo relatos en los que, por un lado, se narra la amenaza a la imagen de un hablante y, por otro, esta trata de ser reparada.

Así pues, con este estudio, se ha pretendido realizar una aproximación general al fenómeno de la reparación discursiva, con especial atención a su coocurrencia con casos de atenuación o intensificación fónicas. En vista a otras futuras investigaciones, sería interesante observar la frecuencia de estas reparaciones discursivas de imagen y su coaparición con factores fónicos, en otras variedades del español ya que este estudio se ha centrado únicamente en la variedad peninsular.

\section{AGRADECIMIENTOS}

Esta investigación ha sido posible gracias a la subvención del proyecto de investigación Es.VaG.Atenuación (La atenuación pragmática en su variación genérica: 
géneros discursivos escritos y orales en el español de España y América; Ministerio de Economía y Competitividad de España, ref. FFI2016-75249-P).

\section{REFERENCIAS BIBLIOGRÁFICAS}

Albelda Marco, Marta. 2004. «La intensificación pragmática y su reflejo a través de la prosodia». En Actas del V Congreso de Lingüistica General: León 5-8 de marzo de 2002, 199-210.

—. 2007. La intensificación como categoría pragmática: revisión y propuesta. Frankfurt: Peter Lang.

—. 2013. «La atenuación: tipos y estrategias». En El español de Valencia: estudio sociolingüístico, ed. José Ramón Gómez Molina. Berna: Peter Lang, 315-43.

—. 2014. «Ficha metodológica para el análisis pragmático de la atenuación en corpus discursivos del español (ES.POR.ATENUACIÓN)». Oralia: Análisis del discurso oral, 17: 7-62.

Asiko Ambuyo, Beverline, Florence Ngesa Indede y Peter Ndichu Karanja. 2011. «Face Threatening Acts and Standing Orders: 'Politeness' or 'politics' in the Question Time Discussions of the Kenyan Parliament». International Journal of Humanities and Social Science, 1(9): 209-18.

Benoit, William L. 1997. «Image repair discourse and crisis communication». Public Relations Review, 23(2): 177-86.

Boersma, Paul y David Weenink. 2017. «Praat: doing phonetics by computer». http://www.praat.org/.

Briz Gómez, Antonio y Marta Albelda Marco. 2013. «Una propuesta teórica y metodológica para el análisis de la atenuación lingüística en español y portugués. La base de un proyecto en común (ES.POR. ATENUACIÓN)». Onomazein, 28(2): 288-319.

Brown, Gillian. 1990. Listening to Spoken English. London: Longman.

Brown, Gillian, Karen L Currie y Joanne Kenworthy. 1985. Croom Helm linguistics series. Questions of intonation. Londres: Croom Helm.

Cabedo Nebot, Adrián y Salvador Pons Bordería. 2013. Corpus Val.Es.Co 2.0. http://www.valesco.es.

Compton, Josh. 2016. «Sorry sorries: Image repair after regretted apologies». Public Relations Review, 42(2): 353-58.

Estellés Arguedas, Maria y Adrián Cabedo Nebot. 2017. «La atenuación fónica en entrevistas (proyecto PRESEEA) y en conversaciones (corpus Val.Es.Co): un estudio de campo». Linred: 1-18.

Fernández Planas, Ana María et al. 2002. «Umbrales tonales en español peninsular». En Actas del II Congreso de Fonética Experimental, ed. J. Díaz García. Sevilla: Universidad de Sevilla, 272-78.

Figueras Bates, Carolina. 2018. «Atenuación, género discursivo e imagen». Spanish in Context, 15(2): 260-82.

Glynn, Dylan. 2010. Lexical Fields, Grammatical Constructions, and Synonymy. A study in usage-based Cognitive Semantics. Berlin: Mouton de Gruyter.

—. 2014a. «Correspondence analysis: Exploring data and identifying patterns». En Corpus methods for semantics: Quantitative studies in polysemy and synonymy, eds. Dylan Glynn y Justina A. Robinson. Amsterdam: John Benjamins, 443-485.

—. 2014b. «Techniques and tools: Corpus methods and statistics for semantics». En Corpus methods for semantics: Quantitative studies in polysemy and synonymy, eds. Dylan Glynn y Justina A. Robinson. Amsterdam: John Benjamins, 307-341. 
Goffman, Erving. 1967. «Interaction Ritual». New York, 48: 282.

Gómez Sánchez, María Elena. 2004. «Cortesía lingüística: un ejemplo de equilibrio y reparación de la imagen en las tertulias radiofónicas». Español actual: Revista de español vivo, 81: 41-46.

Greenacre, Michael. 2007. Correspondence Analysis in Practice. Londres: CRC Press.

Grice, Paul. 1991. «Lógica y conversación». En Búsqueda del significado, ed. Luis Valdés. Murcia: Tecnos, 511-30.

Hernández Flores, Nieves. 2004. «La cortesía como búsqueda del equilibrio de la imagen social». En Pragmática sociocultural: estudios sobre el discurso de cortesía en español, eds. Diana Bravo y Antonio Briz Gómez. Barcelona: Ariel, 95-108.

—. 2015. «La actividad de imagen en el discurso oral: localización y delimitación en la interacción comunicativa». En Perspectivas socio-pragmáticas y socio-culturales del análisis del discurso, eds. Diana Bravo y María Bernal. Buenos Aires: Dunken, 23-48.

Hidalgo Navarro, Antonio. 2009. «Modalización (des)cortés y prosodia: estado de la cuestión en el ámbito hispánico». Boletín de filología: (Universidad de Chile), 44(1): 161-95.

—. 2015. «Prosodia y partículas discursivas: sobre las funciones de atenuación, intensificación como valores (des)corteses en los marcadores conversacionales». Círculo de lingüística aplicada a la comunicación, 62: 76-104.

Izre'el, Shlomo y Amina Mettouchi. 2015. «Representation of speech in CorpAfroAs: Transcriptional strategies and prosodic units». En Corpus-based Studies of Lesser-described Languages. The CorpAfroAs corpus of spoken AfroAsiatic languages, Amsterdam / Philadelphia: John Benjamins Publishing Company, 1341.

Lê, Sébastien, Julie Josse y Fancois Husson. 2008. «FactoMineR». Journal of Statistical Software, 25(1): 1-18. http://www.jstatsoft.org/v25/i01.

López Serena, Araceli. 2011. «¿Es empírico el estudio de la (des)cortesía verbal? El estatus epistemológico de la lingüística de la (des)cortesía». En Aproximaciones a la (des)cortesía verbal en español, Berlin: Peter Lang, 425-42.

Manchón, Lluís Mas. 2011. «La intensidad en la noticia hablada en televisión». Estudios de fonética experimental, 20: 71-112.

Marrero Aguiar, Victoria. 2008. «La fonética perceptiva: trascendencia lingüística de mecanismos neuropsicofisiológicos». Estudios de fonética experimental, 17: 20745.

Max Planck Institute. 2017. «ELAN (Version 5.2)». https://tla.mpi.nl/tools/tlatools/elan/.

Moore, David S. y George P. McCabe. 1999. Introduction to the practice of statistics. W.H. Freeman.

Quilis, Antonio. 1981. Fonética acústica de la lengua española. Madrid: Gredos.

R Development Core Team. 2017. «R Software». R: A Language and Environment for Statistical Computing.

Rojas Avendaño, Darcy, María Alejandra Blondet Serfaty y Alexandra Álvarez Muro. 2014. «Configuración tonal de la atenuación en el habla de Mérida». Lengua y Habla, 18: 93-106.

Roseano, Paolo y Anna Maria Fernàndez Planas. 2013. «Transcripció fonètica i fonològica de l'entonació: una proposta d'etiquetatge automàtic». Estudios de fonética experimental, 22: 275-332.

Spencer-Oatey, Helen. 2007. «Theories of identity and the analysis of face». Journal of Pragmatics, 39(4): 639-56. 
Vela Delfa, Cristina. 2016. «Actividades de imagen en el género discursivo de la canción protesta: un análisis desde la teoría de la cortesía». Analecta Malacitana, 40: 14763.

Villalba, Cristina. 2016. Actividades de imagen, atenuación e impersonalidad en los juicios orales. València: Universitat de València.

Wichmann, Anne. 2000. Studies in language and linguistics Intonation in text and discourse: beginnings, middles, and ends. New York: Longman. 